\title{
A disposable bedpan system using an improved disposal unit and self-supporting bedpans
}

\author{
G. L. GIBSON
}

From the Regional Public Health Laboratory, Leeds

SYNOPSIS A system using totally disposable self-supporting bedpans requiring no carrier was examined in use in two hospitals. The bedpans and their contents were disposed of by destruction and flushing to waste carried out in a modified Haigh Sluicemaster disposal unit. This incorporates a positively closing and locking lid with refinements to avoid the lid slamming and has effective safety devices.

The new bedpans and the improved disposal units reduce the risk of transfer and dispersal of pathogenic organisms to an acceptable level in ward and sluice room.

When disposable bedpan systems were examined previously a number of defects were reported (Gibson, 1973). The use of bedpans which require a plastic carrier to support them was criticized as a potential infection risk and two bedpan disposal units, Haigh Sluicemaster and the J.M.L. Clinimatic, were found to have certain deficiencies and to involve bacteriological hazards in use.

One manufacturer (Haigh Hygiene Ltd has now) offered a modified bedpan destructor and this has been examined in use together with totally disposable self-supporting bedpans manufactured by Van Gelder Papier Ltd.

The modifications to the Haigh Sluicemaster include several significant improvements. The chamber is now fitted with a positive-closing and locking lid. This removes the risk of splashing during the cycle of bedpan destruction and by its close fit avoids wetting of the rim of the chamber. This, in turn, very appreciably reduces the aerosol and dispersal during opening and closing. The locking device is protected by a micro-switch which cuts off the motor if the handle is moved, before any opening movement of the lid is possible. A further refinement is the fitting of a pneumatic device on the foot-pedal linkage to the chamber lid which dampens the previously coarse movement and avoids the slamming action which tended to disperse water and particles widely. The total result of these modifications is to reduce the escape of aerosol and particles from the chamber to insignificant levels.

The improvement is enhanced by the removal of the vent pipe from the chamber. This means that any Received for publication 29 August 1973. potential risk of bacterial spread from the vent itself is ended and at the same time it allows a vacuum to build up in the chamber during the cycle so that any pressure at the rim of the lid is inwards. A valve on the water supply pipe between the pump and the chamber allows air to enter the system when the cycle is over and the water pressure falls. The lid may then be opened, the small terminal vacuum being overcome by a simple device on the locking handle. The total cycle time is 110 seconds.

A diagrammatic representation of the modified machine is shown in the figure. All the safety devices previously described on the unmodified machine are still fitted. The drain pressure 'cut-out' which cuts off the cycle if the drain is blocked tended in previous models not to operate until a considerable head of water built up in the chamber because of the escape of air via the lid and the vent. In the modified model the 'cut-out' acts very rapidly when the drain is blocked as only a minimal escape of air is possible.

Tests of the escape of chamber contents during the cycle were carried out in a similar manner to those described in the previous investigation. Petri dishes containing blood agar were exposed in a ring, face downwards, overhanging the rim of the lid. In tests during running the petri dishes were laid over the join of the lid and the cabinet top. Eleven plates were required and were numbered 1-11 clockwise starting at the hinge. During tests of opening and closing, the 11 petri dishes were firmly attached with adhesive tape with the greater part of their exposed downward facing surface overlapping the rim. Any escape of aerosol or particles from the chamber impinged on. 


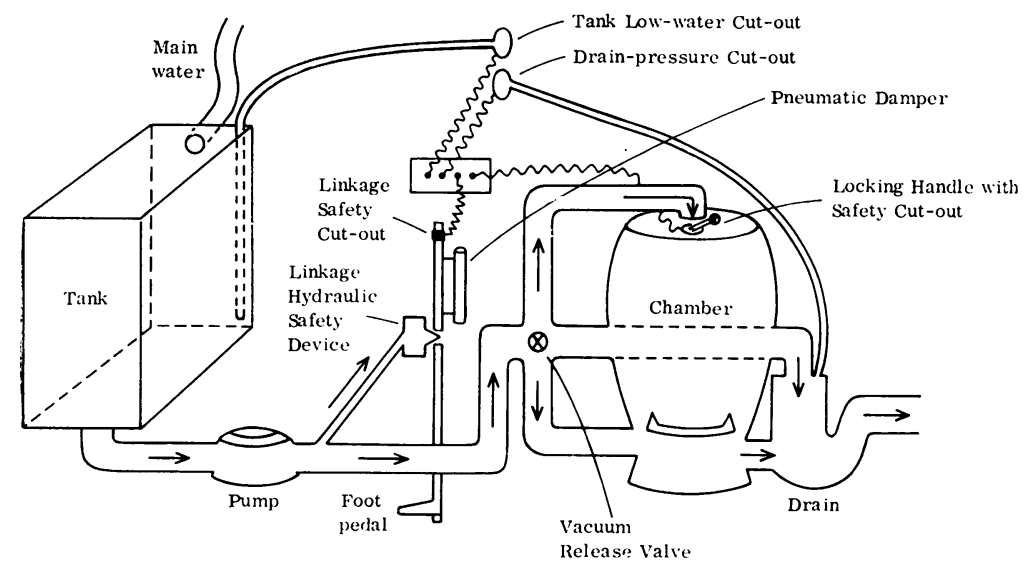

Table I Opening lid

\begin{tabular}{|c|c|c|c|c|c|c|c|c|c|c|c|c|c|}
\hline \multicolumn{2}{|c|}{ Contents of Bedpan } & \multicolumn{12}{|c|}{ Growth on Blood Agar Plates ${ }^{1}$} \\
\hline Faeces & Urine & 1 & 2 & 3 & 4 & 5 & 6 & 7 & 8 & 9 & 10 & 11 & \\
\hline 2 & 0 & - & - & - & - & - & - & \pm & - & - & - & - & \\
\hline 2 & 0 & - & - & - & \pm & \pm & - & - & \pm & - & - & - & ชి \\
\hline 2 & 0 & - & - & - & - & \pm & - & - & - & - & - & - & 무 \\
\hline 1 & 1 & - & \pm & - & + & \pm & - & - & - & - & - & - & $\leqq$ \\
\hline 2 & 0 & - & $=$ & - & - & $=$ & - & \pm & - & - & - & - & $\overline{\mathrm{Q}}$ \\
\hline 1 & 1 & - & \pm & - & - & - & - & \pm & - & - & - & - & $\tau$ \\
\hline
\end{tabular}

\section{Table I Opening lid}

\begin{tabular}{|c|c|c|c|c|c|c|c|c|c|c|c|c|}
\hline \multicolumn{2}{|c|}{ Contents of Bedpan } & \multicolumn{11}{|c|}{ Growth on Blood Agar Plates } \\
\hline Faeces & Urine & 1 & 2 & 3 & 4 & 5 & 6 & 7 & 8 & 9 & 10 & 11 \\
\hline 2 & 0 & \pm & - & - & - & \pm & \pm & - & + & + & \pm & + \\
\hline 2 & 0 & \pm & - & - & \pm & \pm & - & + & + & \pm & - & \pm \\
\hline 2 & 0 & - & - & - & + & \pm & - & \pm & - & - & - & - \\
\hline 1 & 1 & - & \pm & - & - & \pm & - & - & - & \pm & - & - \\
\hline 2 & 0 & - & - & - & - & + & - & \pm & - & - & \pm & - \\
\hline 1 & 1 & \pm & - & \pm & - & \pm & - & - & - & - & \pm & - \\
\hline
\end{tabular}

Table II Shutting lid

\begin{tabular}{|c|c|c|c|c|c|c|c|c|c|c|c|c|}
\hline \multicolumn{2}{|c|}{ Contents of Bedpan } & \multicolumn{11}{|c|}{ Growth on Blood Agar Plates } \\
\hline Faeces & Urine & 1 & 2 & 3 & 4 & 5 & 6 & 7 & 8 & 9 & 10 & 11 \\
\hline 2 & 0 & - & - & - & - & - & - & - & - & - & - & - \\
\hline 2 & 0 & \pm & - & - & - & - & - & - & - & - & - & - \\
\hline 2 & 0 & - & - & \pm & - & - & - & - & - & - & - & - \\
\hline 1 & 1 & - & - & - & - & - & - & - & - & - & - & - \\
\hline 2 & 0 & - & - & - & - & - & - & \pm & - & - & - & - \\
\hline 1 & 1 & - & - & - & - & - & - & - & - & - & & - \\
\hline
\end{tabular}

Table III During run

$-=$ no growth, $\pm=1-4$ colonies, $+=5-19$ colonies

${ }^{1}$ Plates are numbered 1-11 clockwise from the hinge round the lid. 
the blood agar. The machine was loaded with bedpans containing faeces $(F)$ and urine (U). In contrast with the findings of considerable contamination in the unmodified machines previously tested, no evidence of escape of organisms could be shown during running and only very minimal contamination during opening and closing. Surface colony counts were arbitrarily divided as follows: $-=$ no growth, $\pm=1-4$ colonies per plate, and $+=5-19$ colonies per plate. No plate showed more than 19 colonies. Results may be seen in tables I, II, and III. It was not possible to repeat the disturbing demonstration of the gross escape of material which took place on deliberately forcing open the lid during running, as this was no longer possible in the modified machine.

In further tests the rim of the chamber was thoroughly cleaned with methylated spirit, allowed to dry, the machine loaded carefully, and the lid closed. Swabs were then taken before the run, after the run, and after opening the lid, plated on blood agar, and incubated at $37^{\circ} \mathrm{C}$ for 18 hours aerobically. There was no macroscopic evidence at any time of contamination of the top of the cabinet or of its interior and the cultures obtained from the rim swabs were of scanty aerobic spore bearers only.

Thirteen hundred totally disposable self-supporting bedpans were made available, 1000 of these being assessed in use in a geriatric ward in one hospital and 300 in an orthopaedic ward in a second hospital. These pans, unlike the type commonly used, are constructed in such a way as to support the weight of the patient by themselves. Instead of being a simple lining designed to sit inside a bedpan-shaped carrier of plastic or fibre-glass they have a strong outer rim forming a surrounding wall which allows the actual pan itself to bear the weight. The pan is approximately ovoid giving a wider opening than the bedpan-linings offer, but a narrower rim, and the texture is noticeably finer and smoother. The pans weigh $180 \mathrm{~g}$ as opposed to the lining's weight of $95 \mathrm{~g}$. This of course means that there will be a greater weight of papier maché involved in each bedpan use. The cost of each bedpan should be not more than twice that of the old bedpan liner. The makers of the machine tested advise a limit of two of the new bedpans per cycle although the machine coped well with three. The cycle time of 110 seconds is not prolonged and should be acceptable in any intelligently run ward. The major advantage of the new bedpans is of course the elimination of the dangers of the spread of infection by the bedpan carriers. The nursing staff is relieved of the task of cleaning and disinfecting them, and there is no possibility of a contaminated article being offered to the patient.
The bedpans were uniformly well received by nurses and patients in the two hospitals. The nurses appreciated not having the work and worry of dealing with the carriers and the majority of patients accepted the new pans as offering a wider, more comfortable seat on a smoother rim. Some, however, preferred the broader rim of the bedpan-liner in its fibre-glass support but even this group did not object to the new pans. Their shape may be further modified in future by slimming down the outer wall which will facilitate loading the bedpan disposal unit.

\section{Discussion}

The problem of the most suitable and acceptable system of providing bedpans and urinals to patients continues to exercise many of those responsible. Any system must be acceptable aesthetically and on grounds of comfort to the patient. It must be simple, reliable, not unpleasant to the nurse, and should be economical in capital, revenue, and labour. Above all it must be bacteriologically safe in two separate areas, the patient's bed and the ward sluice room.

Stainless steel non-disposable bedpans meet some of these criteria provided that reliable cleaning and sterilization are possible. Thorough cleaning followed by disinfection may be acceptable but whatever method is used requires expensive installations and services. Basically there remains the undesirable necessity to return the bedpan to the bed of the patient himself or his neighbour. The sterilization or disinfection process involving heat has got to be guaranteed in repeated use and must not be slow or inefficient. Bedpan washers not served by steam cannot be relied upon to disinfect rapidly and repeatedly. In these circumstances there must be a potential risk to both patient and nurse.

Disposable bedpan liners of the type commonly available hitherto require little capital, more revenue, and some labour. While the bedpan liner is not returned to the patient's bed the carrier or support is. The nurse is required to cope with cleaning and disinfecting the carrier which, in practice, is contaminated by faeces on many occasions and must invariably be contaminated by the patient's bedding. The patient then is put at some potential risk, and may even be presented with an obviously offensive article. In addition the unmodified bedpan destructors in the sluice room present bacteriological hazards to the nurse which can be of an unacceptable level.

Totally disposable self-supporting bedpans present no hazard to the patient whatsoever, as on each occasion he is presented with a fresh article. The modified bedpan destructor now described does not offer appreciable dangers to the nurse in the sluice. 
room. The level of contamination obtained in tests on the modified machines is very greatly reduced in comparison with the levels found in the unmodified machines examined previously, where gross escape of organisms, aerosol, and particles was demonstrated, particularly during shutting the lid and also during running. The rim of the chamber and the surface of the casing of the machine remain almost entirely dry and free of contamination in contrast with the previous findings. The safety devices are greatly improved, especially the mechanism to avoid opening the lid during running. The vent pipe with its potential danger has been removed and the coarse action in shutting the lid eliminated. Capital outlay is relatively low although revenue costs are greater. Labour is cut to a minimum and the system $\stackrel{0}{\vec{F}}$ is very well received by both nurse and patient.

The modifications to the bedpan disposal unit, together with the use of totally disposable self-supporting bedpans, offer a system which should bee acceptable in all types of wards.

I wish to thank the engineering staff of York $A \stackrel{\varnothing}{\varrho}$ Group and Leeds B Group of Hospitals and thes nursing staff of Yearsley Bridge and Marguerite $\overrightarrow{0}$ Hepton Hospitals for their help. I am grateful to Mrs I. R. Lynn for secretarial assistance.

\section{Reference}

Gibson, G. L. (1973). Bacteriological hazards of disposable bedpari systems. J. clin. Path., 26, 146-153. 